\title{
MicroRNA-135b regulates the stability of PTEN and promotes glycolysis by targeting USP13 in human colorectal cancers
}

\author{
SHIJUN XIANG ${ }^{*}$, JIAQING FANG $^{2 *}$, SHUYUN WANG $^{1^{*}}$, BIAO DENG $^{1}$ and LIN ZHU ${ }^{1}$ \\ ${ }^{1}$ Department of General Surgery, Shanghai First People's Hospital, Shanghai Jiao Tong University, Shanghai 200080; \\ ${ }^{2}$ Department of Gastroenterology, Changzheng Hospital, Second Military Medical University, Shanghai 200003, P.R. China
}

Received September 2, 2014; Accepted November 30, 2014

DOI: 10.3892/or.2014.3694

\begin{abstract}
Dysregulation of microRNAs has been reported to be involved in the progression of human colorectal cancers (CRCs). Loss of the adenomatous polyposis coli (APC) gene is a common initiating event in CRCs. PTEN inactivation by mutation or allelic loss also occurs in CRCs. miR-135b was reported to be upregulated in CRCs and its overexpression was due to APC/ $\beta$-catenin and PTEN/PI3K pathway deregulation. APC was proven to be a target of miR-135b and forms a feedback loop with miR-135b. In the present study, we found that ubiquitin-specific peptidase 13 (USP13) was a target of miR-135b. miR-135b downregulated the expression of USP13, and reduced the stability of PTEN. miR-135b promoted cell proliferation and glycolysis that could be reversed by the overexpression of USP13 or PTEN. Moreover, knockdown of USP13 upregulated the levels of endogenous miR-135b, but not those in CRC cells with PTEN mutation. The results showed positive feedback loops between miR-135b and PTEN inactivation in CRCs.
\end{abstract}

\section{Introduction}

Colorectal cancer (CRC) is the third most common malignant neoplasm worldwide and the fourth most common cause of cancer mortality $(1,2)$. It affects health and life span (3). Thus, the understanding of the molecular pathology of CRC is crucial in the identifications of treatments. Accumulation of multiple genetic alterations transforms normal colonic epithelial cells to adenocarcinoma cells (4-6). Genetic alterations

Correspondence to: Dr Biao Deng or Professor Lin Zhu, Department of General Surgery, Shanghai First People's Hospital, Shanghai Jiao Tong University, 100 Haining Road, Shanghai 200080, P.R. China

E-mail: derikdeng@163.com

E-mail: zhulin5995@126.com

\section{${ }^{*}$ Contributed equally}

Key words: microRNA-135b, colorectal cancers, PTEN, USP13, glycolysis are associated with the activation of proto-oncogenes (KRAS and $B R A F)$ (7-11) and the inactivation of tumor suppression genes (APC, DCC/SMAD4, TP53 and PTEN) (12-15). The APC $/ \beta$-catenin pathway plays a key role in CRC. Adenomatous polyposis coli $(A P C)$ is the most common initial gene mutated in CRC and the majority of APC mutations are either frameshift or nonsense mutations leading to the synthesis of truncated proteins (16). PTEN is known as a tumor suppressor through negative regulation of the PI3K/Akt pathway $(17,18)$. Allele loss close to the PTEN locus has been reported in $30 \%$ of sporadic CRC (19).

MicroRNAs (miRNAs) are small, regulatory, non-coding RNA molecules that lead to silencing of their genes via mRNA degradation or preventing mRNA from being translated (20). miRNAs have been involved in the regulation of a variety of cell processes, such as DNA repair (21-23), metastasis and metabolism (24-27). Thus, miRNAs function as oncogenes or tumor suppressors. Dysregulation of miRNAs has been involved in the progression of CRC $(28,29)$. Recent studies have examined the expression patterns of miRNA in CRC and identified a variety of miRNAs that were dysregulated in CRC $(30,31)$.

miR-135b was reported to function as a key molecule downstream of the oncogenic pathway involved in cancer progression (32-35). miR-135b was upregulated in CRCs and its overexpression was due to APC/ $\beta$-catenin and PTEN/PI3K pathway deregulation (36). In the present study, we showed that miR-135b regulates the stability of PTEN protein. Deubiquitinating enzyme USP13, which stabilizes the PTEN protein through direct binding and deubiquitinating of PTEN, was proven as as a target of miR-135b. Overexpression of miR-135b downregulates the expression of ubiquitin-specific peptidase 13 (USP13) and reduces the stability of PTEN. miR-135b also promotes cell proliferation and glycolysis, which could be reversed by the overexpression of USP13/PTEN. Moreover, knockdown of USP13 upregulates the levels of endogenous miR-135b. These results revealed positive feedback loops between miR-135b and PTEN inactivation in CRCs.

\section{Materials and methods}

Reagents. Cycloheximide (CHX) was purchased from Sigma-Aldrich (St. Louis, MO, USA). Anti-PTEN and anti-USP13 were purchased from Millipore (Bedford, MA, 
USA). Anti-p-AKT (Ser 473), anti-AKT and anti- $\beta$-actin were purchased from Cell Signaling Technology, Inc. (Beverly, MA, USA). The real-time PCR Master mix kit was purchased from Takara (Dalian, China).

Cell cultures and transfections. HCT-116 CRC cell lines were purchased from the American Type Culture Collection (ATCC, Dallas, TX, USA). Caco2, CO-115 and 293T cell lines were purchased from the Type Culture Collection of the Chinese Academy of Sciences (Shanghai, China). HCT-116, Caco2, CO-115 and 293T cells were cultured in DMEM (Invitrogen, Carlsbad, CA, USA) supplemented with $10 \%$ fetal bovine serum (Hyclone, Logan, UT, USA), penicillin $(100 \mathrm{U} / \mathrm{ml})$ and streptomycin $(100 \mu \mathrm{g} / \mathrm{ml})$ in a humidified atmosphere of $5 \% \mathrm{CO}_{2}$ at $37^{\circ} \mathrm{C}$. The cells were examined for mycoplasma contamination periodically and were always found to be negative. Cell transfections were performed using Lipofectamine 2000 (Invitrogen) following the manufacturer's instructions.

RT-qPCR for mature miR-135b and PTEN. Total RNA was extracted from cell lines using Trizol (Invitrogen). RT-qPCR of miR-135b was performed using TaqMan miRNA reverse transcription kit (Applied Biosystems, Foster City, CA, USA) and TaqMan human miRNA assay (Assay ID: RNU6B, 001093; miR-135b, 002261). For detection of the mRNAs of PTEN, total RNA was extracted by TRIzol (Invitrogen) and subjected to reverse transcription using the PrimeScript II first-strand cDNA synthesis kit (Takara) according to the manufacturer's instructions. The primers for human PTEN were: forward, 5'-CAAGATGATGTTTGAAACTATTCCAATG-3' and reverse, 5'-CCTTTAGCTGGCAGACCACAA-3'. RT-qPCR was performed with the 7500 Fast real-time PCR system (Applied Biosystems). miR-135b expression was normalized to that of RNU6B. PTEN expression was normalized to GAPDH. Reverse-transcriptase (RT) reactions, including no-template controls and RT minus controls, were run in a GeneAmp PCR 9700 Thermocycler (Applied Biosystems). After the Ct values of the target genes were subtracted by that of the internal control RNU6B/GAPDH, the relative expression level of each target gene was obtained using the $2^{-\Delta \Delta \mathrm{Ct}}$ method.

Metabolism assays. Glucose consumption was measured in the cell lysates with a Glucose uptake colorimetric assay kit (BioVision, San Francisco, CA, USA). The extracellular lactate was measured in the medium using a lactate assay kit (BioVision) according to the manufacturer's instructions.

Construction of plasmids. For luciferase reporter experiments, the 3'-UTR segment of the USP13 gene was amplified by PCR using human cDNA as templates and inserted into the pGL3-control vector (Promega, Madison, WI, USA). A mutant construct was generated from the perfect complementary site using the Quik change site-directed mutagenesis kit (Stratagene, La Jolla, CA, USA).

Viral production and infection. MiRNASelect ${ }^{\mathrm{TM}}$ pEPmiR-Null control lentiviral vector and miRNASelect ${ }^{\mathrm{TM}}$ pEP-hsa-mir-135b lentiviral expression vector were obtained from Cell Biolabs (San Diego, CA, USA). Human miRNA inhibitors against human miR-135b in the lentiviral vector were obtained from OriGene (Rockville, MD, USA). Expression lentiviral vector for shRNA against USP13 was constructed into a pLKO.1 vector. The target sequences were: 5'-CGATTTAAATAGCGACGATTA-3' (sh-13-1) and 5'-GCC AGTATCTAAATATGCCAA-3' (sh-13-2). Negative control vector containing scrambled shRNA was obtained from Addgene (Cambridge, MA, USA). pLenti-C-Myc-DDK tagged USP13 or PTEN lentiviral expression vector was purchased from OriGene. The plasmids were prepared with plasmid maxi kit and transfected in $293 \mathrm{~T}$ cells (Invitrogen) with the Lipofectamine ${ }^{\mathrm{TM}} 2000$ to produce lentiviral particles. The cells were infected with lentiviral particles for $2 \mathrm{~h}$. The cells were then selected with $2.5 \mathrm{mg} / \mathrm{ml}$ puromycin for $72 \mathrm{~h}$.

Western blot analysis. Cells were lysed in a RIPA buffer containing complete protease/phosphatase inhibitor cocktail (Cell Signaling Technology, Inc.). The lysate was centrifuged at $12,000 \mathrm{rpm}$ for $15 \mathrm{~min}$ at $4^{\circ} \mathrm{C}$ and the supernatant was collected. Protein concentration was determined by the Bradford protein assay. The proteins were separated by SDS-PAGE gel electrophoresis and then transferred onto PVDF membranes (Millipore). The membranes were blocked with $10 \%$ BSA in TBST for $1 \mathrm{~h}$ and incubated with antibodies overnight at $4^{\circ} \mathrm{C}$. After washing with TBST three times, the membranes were probed with HRP-conjugated secondary antibodies in TBST for $1 \mathrm{~h}$ at room temperature, then washed with PBST three times. The immunobinding signals were detected by a chemiluminescence method (ECL, Millipore).

Luciferase activity assay. To directly evaluate the effect of miR-135b on USP13, the luciferase assay was used. Stable CRC cell lines that constitutively express human miR-135b were generated. The cells were transfected with firefly luciferase reporter vector and the control vector pRL-CMV (coding for Renilla luciferase, Promega) or plus miR-135b inhibitor lentiviral particles. Forty-eight hours after transfection, firefly and Renilla luciferase activities were measured using the Dual-Luciferase reporter assay system (Promega), and the relative reporter activity was normalized to Renilla luciferase activity. Each assay was repeated in six independent experiments.

Cell proliferation analysis. Cell proliferation was examined by using a Cell Counting kit-8 (Dojindo, Kumamoto, Japan). The cells were plated in 96 -well plates at $2 \times 10^{3}$ cells/well and cultured in growth medium for $24 \mathrm{~h}$. After $24 \mathrm{~h}, \mathrm{CCK}-8$ (10 $\mu \mathrm{l}$ ) was added to each well containing $100 \mu \mathrm{l}$ of DMEM medium. The plate was incubated for $2 \mathrm{~h}$ at $37^{\circ} \mathrm{C}$. Absorbance was measured at $450 \mathrm{~nm}$ using a microplate reader (Tecan, Switzerland).

Statistical analysis. Data were analysed using the Student's t-test. $\mathrm{P}<0.05$ was considered to indicate a statistically significant difference.

\section{Results}

miR-135b upregulates the stability of PTEN protein. $\mathrm{miR}-135 \mathrm{~b}$ was reported to be upregulated in CRCs and its overexpression 
A

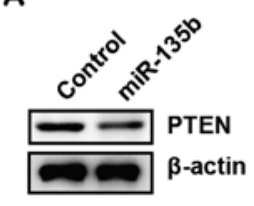

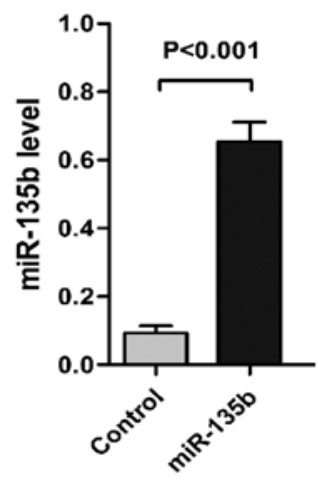

B
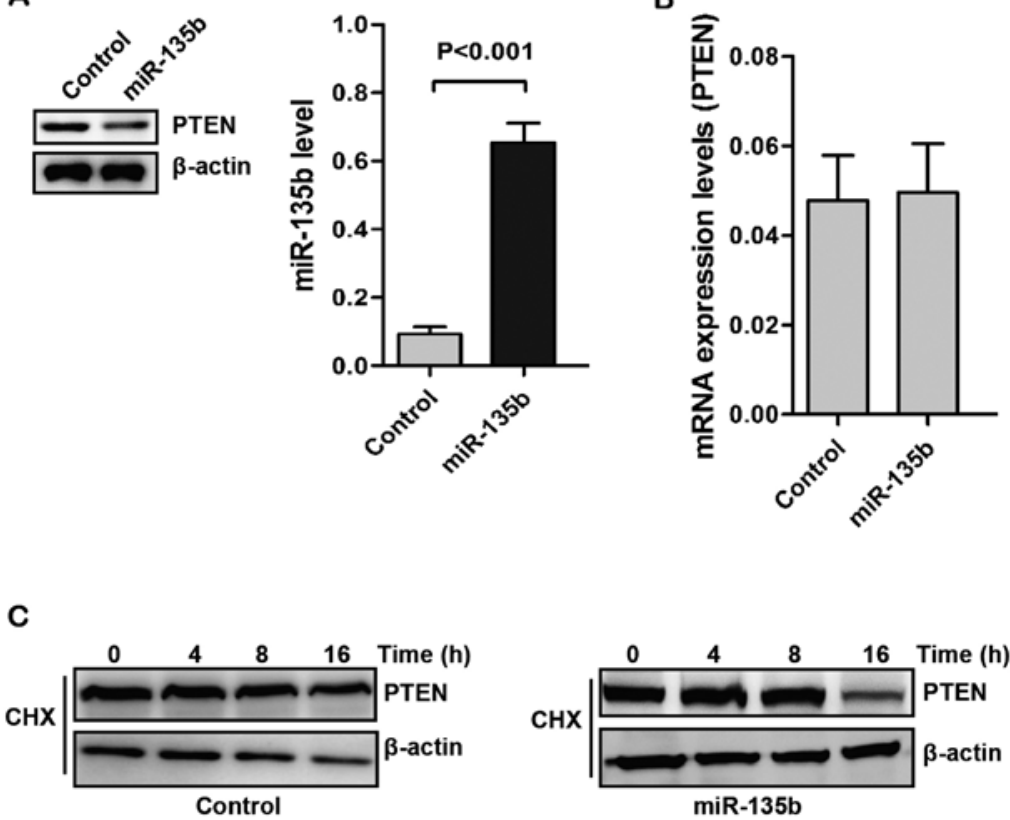

Figure 1. miR-135b upregulates the stability of PTEN protein. (A) Caco2 cells were stably transfected with control vector or miR-135b. The expression of PTEN was analyzed by western blotting (Left). The levels of miR-135b were examined by real-time PCR (Right). (B) The levels of PTEN mRNA were examined by real-time PCR. (C) Caco2 control vector or miR-135b-overexpressing cells were treated without or with $50 \mu \mathrm{g} / \mathrm{ml}$ of cycloheximide (CHX) for the indicated times. PTEN and $\beta$-actin were detected by western blotting. P $<0.001$ vs. control.

was due to loss of APC and PTEN. APC was proven to be a target of miR-135b and formed a feedback loop with miR-135b (36). We determined whether miR-135b affected the levels of PTEN. To examine this potential effect, we generated stable Caco 2 cell lines that constitutively express human miR-135b (Fig. 1A). Overexpression of miR-135b decreased the levels of PTEN protein. We then examined the expression of PTEN mRNA by RT-qPCR. In RT-qPCR analysis, no statistically significant change in PTEN mRNA expression was observed between the control and miR-135b overexpressing groups (Fig. 1B).

The role of ubiquitination in modulating PTEN protein has been identified $(37,38)$. The regulation of PTEN levels by miR-135b may be the results of protein stability regulation. We determined the PTEN stability in Caco2 cells with treatment of cycloheximide (CHX). As shown in Fig. 1C, upregulation of miR-135b decreased the stability of PTEN. These results suggested that miR-135b may regulate the stability of PTEN in post-translational levels.

Targeting of USP13 by $m i R-135 b$. miR-135b is predicted not to target PTEN mRNA. To determine the function of miR-135b in the regulation of PTEN stability we used the computational algorithms of TargetScan (ver. 5.2) for prediction analysis to identify target genes of miR-135b. USP13 is a deubiquitinase that mediates deubiquitination of the target proteins. USP13 was reported to stabilize the PTEN protein by direct binding and deubiquitination of PTEN (39). Along with these observations, reassessment of computational prediction suggested that miR-135b potentially targets USP13 gene (Fig. 2A). Overexpression of miR-135b suppressed USP13 protein expression in Caco 2 cells (Fig. 2B). We also found that overexpression of miR-135b significantly reduced the luciferase activity of plasmids containing the 3'-UTR of USP13 (Fig. 2C).
The putative mutated target sites of USP13 3'-UTR were introduced to further investigate the direct regulatory effect. The reporter assay showed that the effects of miR-135b repression were eliminated when the target sites of USP13 3'-UTR were mutated (Fig. 2D). Moreover, the suppressive effects of miR-135b on the USP13 3'-UTR-carrying luciferase were significantly reduced by the expression of miRNA inhibitor against human miR-135b (Fig. 2E). These results indicated that miR-135b regulated the expression of USP13 through a direct seed sequence interaction.

miR-135b promotes glycolysis. The Warburg effect is defined by an increased glucose uptake via glycolysis as a cellular resource, and is a common phenotype of cancer cells (40). AKT was reported to play a critical role in regulating the Warburg effect (41). USP13 can regulate AKT phosphorylation by stabilizing PTEN protein. Thus, USP13 can suppress glycolysis and cell proliferation (39). We hypothesized that miR-135b might regulate glycolysis through the downregulation of USP13/PTEN. Overexpression of miR-135b increased AKT phosphorylation (Fig. 3A). Additionally, glucose uptake and glycolysis of CRC cells were promoted by miR-135b (Fig. 3B).

miR-135b promotes glycolysis and cell proliferation via downregulation of USP13 or PTEN. To determine whether miR-135b-mediated upregulation of glycolysis depends on USP13, we restored the expression of USP13 in miR-135b stably expressing CRC cells. Glucose uptake and glycolysis in miR-135b-overexpressing CRC cells were decreased with the restoration of USP13 (Fig. 4A). Similarly, overexpression of PTEN decreased glucose uptake and glycolysis (Fig. 4B). Moreover, miR-135b promoted the proliferation of 
A

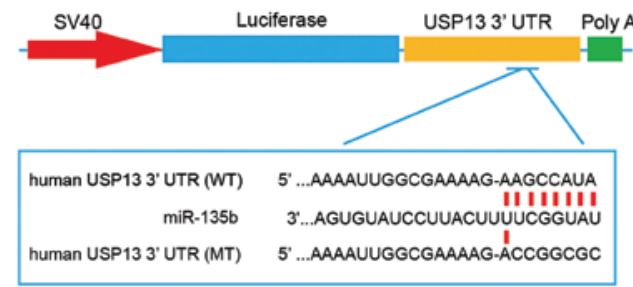

B
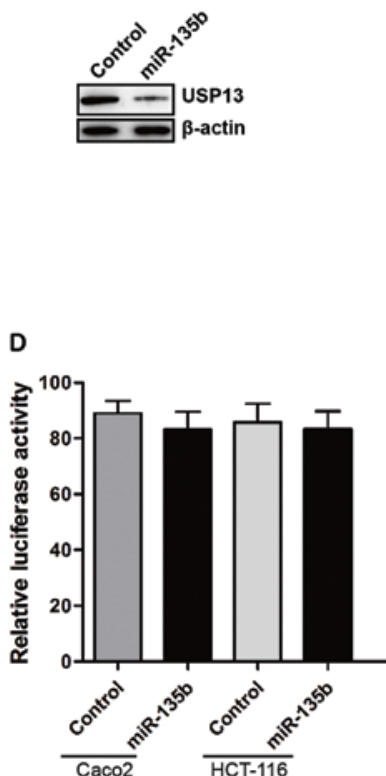

C

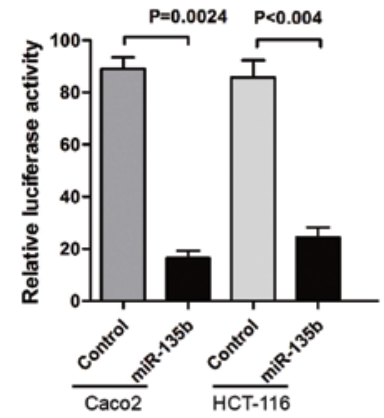

E

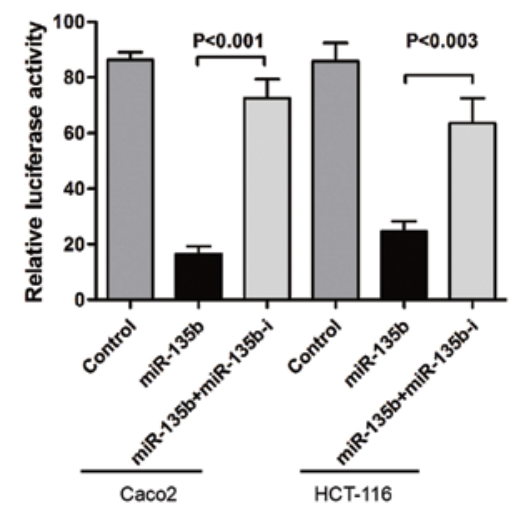

Figure 2. Targeting of USP13 by miR-135b. (A) Recombinant plasmid used in the luciferase assay for miR-135b activity. The 3'-UTR region of the USP13 gene was cloned downstream of the luciferase open reading frame of the pGL3-control plasmid. Sequence alignment between miR-135b and its putative binding site in the USP13 3'-UTR (WT). In the USP13 3'-UTR (MT) construct, the binding site for miR-135b is mutated as indicated. (B) Caco2 cells were stably transfected with control vector or miR-135b. The expression of USP13 was analyzed by western blotting. (C) Stably overexpressing miR-135b inhibited the luciferase activity of the USP13 3'-UTR (WT)-luciferase construct in Caco2 and HCT-116 cells. (D) Stably overexpressing miR-135b did not inhibit the luciferase activity of the USP13 3'-UTR (MT)-luciferase construct in Caco2 and HCT-116 cells. (E) miR-135b inhibitor (miR-135b-i) restored the luciferase activity of the USP13 3'-UTR (WT)-luciferase construct that was inhibited in miR-135b stably overexpressing Caco2 and HCT-116 cells.

A

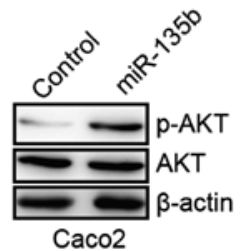

B

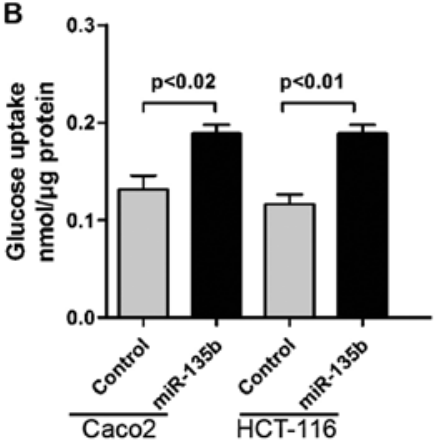

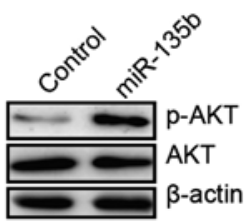

HCT-116

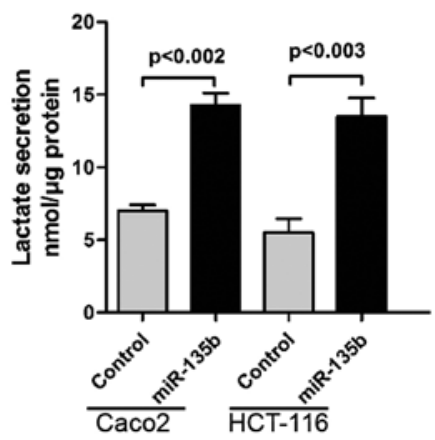

Figure 3. miR-135b promotes glycolysis. (A) Caco2 cells were stably transfected with control vector or miR-135b. The levels of p-AKT, AKT and $\beta$-actin were detected by western blotting. (B) Caco2 and HCT-116 cells were stably transfected with control vector or miR-135b. Glucose uptake and lactate production were measured in medium or lysates and normalized to the total cell protein amount. 

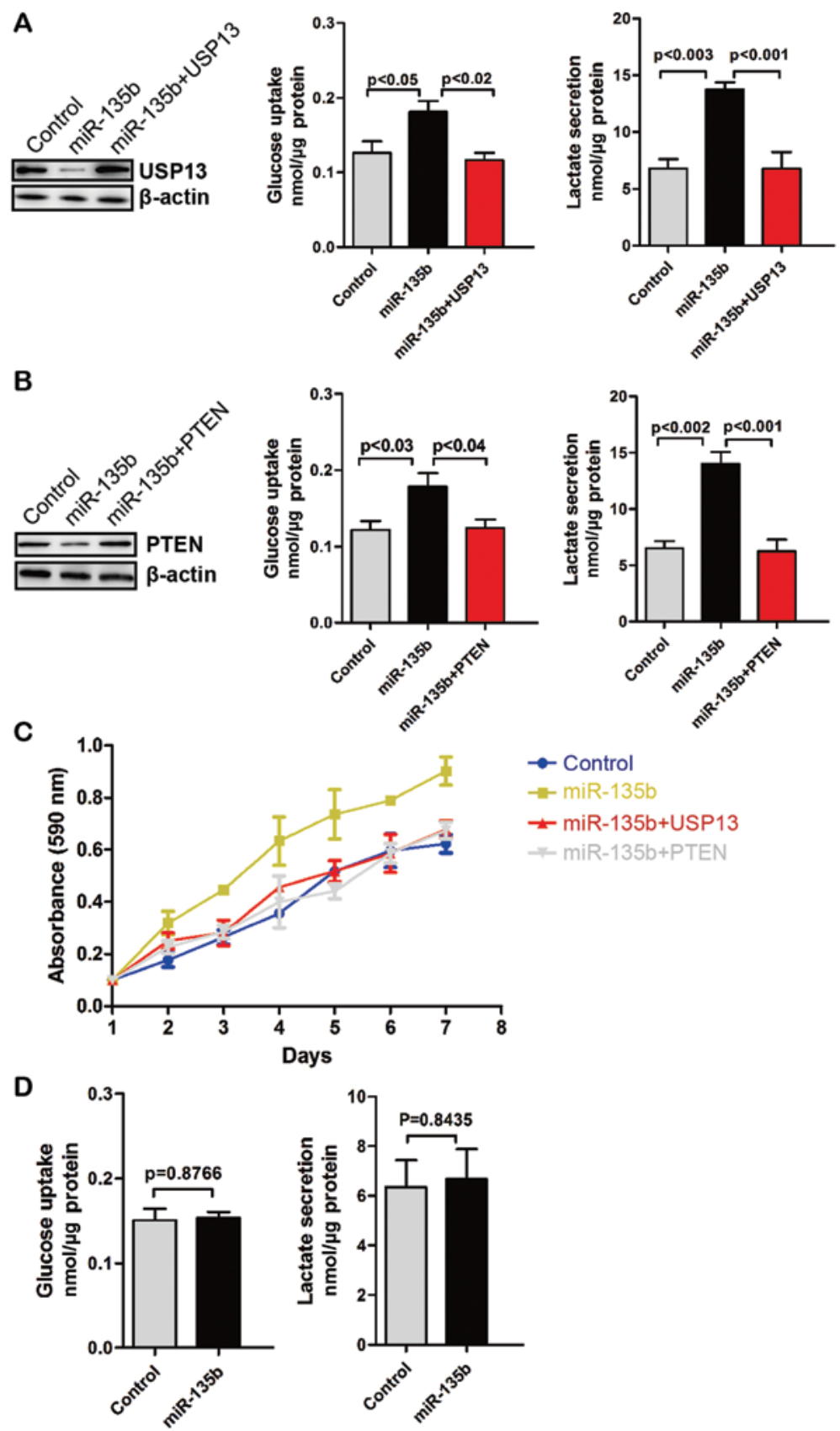

Figure 4. miR-135b promotes glycolysis and cell proliferation via the downregulation of USP13 or PTEN. (A and B) Caco2 and HCT-116 cells were stably transfected with control vector or miR-135b or miR-135b plus USP13/PTEN-overexpressing. The levels of USP13/PTEN and $\beta$-actin were detected by western blotting. Glucose uptake and lactate production were measured in medium or lysates and normalized to total cell protein amount. (C) Caco2 cells were stably transfected with control vector or miR-135b or miR-135b plus USP13/PTEN-overexpressing. The cell proliferation was evaluated by CCK-8. (D) CO-115 cells were stably transfected with control vector or miR-135b. Glucose uptake and lactate production were measured in medium or lysates and normalized to the total cell protein amount.

HCT-116 CRC cells but not with the restoration of USP13 or overexpression of PTEN (Fig. 4C). CO-115 CRC cells have mutations leading to the premature stop codon of PTEN. Thus, we overexpressed miR-135b in CO-115 cells. We found that miR-135b did not affect glucose uptake and glycolysis in CO-115 cells (Fig. 4D). Therefore, miR-135b promotes cell proliferation and glycolysis via the downregulation of USP13 or PTEN.

Knockdown of USP13 increases the levels of endogenous $m i R-135 b$. PTEN is a negative regulator of the PI3K pathway and overexpression of miR-135b partly depends on PI3K pathway. We determined whether the levels of USP13 affect the expression of endogenous miR-135b. Knockdown of USP13 in Caco2 cells decreased the levels of PTEN (Fig. 5A). By contrast, the levels of endogenous miR-135b were significantly increased in USP13-knockdwon Caco2 cells (Fig. 5A). We knocked down the expression of USP13 in CO-115 cells. However, knockdown of USP13 did not affect the levels of endogenous miR-135b in CO-115 cells (Fig. 5B). These results suggested that loss of USP13 upregulates the levels of endogenous miR-135b by reducing the stability of PTEN. 


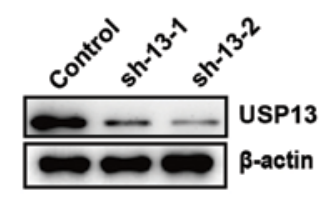

B

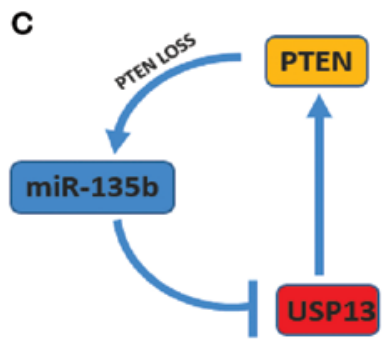

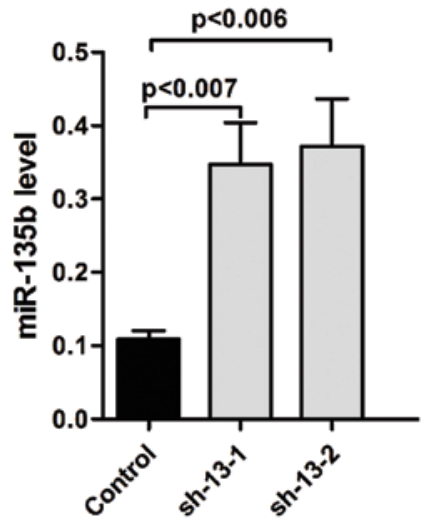

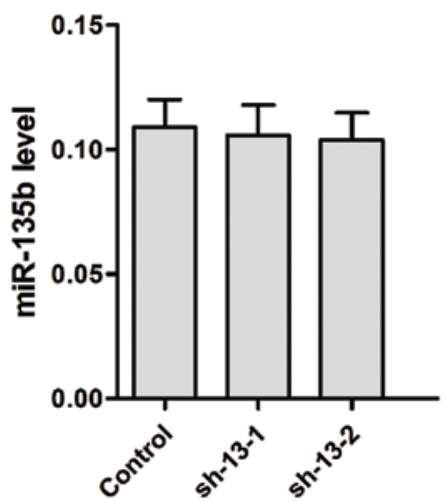

Figure 5. Knockdown of USP13 increases the levels of endogenous miR-135b. (A) The expression of USP13 was analyzed by western blotting in Caco2 cells with stable expression of control-shRNA and shRNA targeting USP13 (Control, sh-13-1 and sh-13-2). The levels of endogenous miR-135b were examined by RT-qPCR. (B) The expression of USP13 was analyzed by western blotting in CO-115 cells with the stable expression of control-shRNA and shRNA targeting USP13 (Control, sh-13-1 and sh-13-2). The levels of endogenous miR-135b were examined by RT-qPCR. (C) Schematic representation of positive feedback loops between miR-135b and PTEN inactivation in CRCs.

\section{Discussion}

As described earlier, miR-135b functions as a key molecule downstream of oncogenic pathways involved in CRC progression (36). miR-135b and its networks are potential therapeutic targets in CRC. The levels of miR-135b in serum were found to be biomarkers for early detection of CRC (42). Xu et al showed that miR-135b is correlated with the clinical parameters of CRC (43). miR-135b was found to be involved in CRC cell invasion (34). Moreover, the overexpression of miR-135b was due to APC/ $\beta$-catenin and PTEN/PI3K pathway deregulation. APC is also the target of miR-135b (36). In the present study, we have shown that miR-135b can regulate the stability of PTEN protein. Deubiquitinating enzyme USP13 can stabilize the PTEN protein by direct binding and deubiquitination of PTEN (39). We used the computational algorithms of TargetScan for prediction analysis to identify target genes of miR-135b and found that USP13 is a potential target of miR-135b (Fig. 2A). Luciferase assay showed that miR-135b significantly reduced the USP13 3'-UTR-carrying luciferase (Fig. 2C). However, the reporter assay showed that the effects of miR-135b repression were eliminated when the target sites of USP13 3'-UTR were mutated (Fig. 2D). Moreover, the suppressive effects of miR-135b on the USP13 3'-UTR-carrying luciferase were significantly reduced by miR-135b inhibitor (Fig. 2E). This result indicates that USP13 is a target of miR-135b.

The Warburg effect is a common phenotype of cancer cells by increasing glucose uptake via glycolysis (44). AKT was reported to play a critical role in regulating the Warburg effect. USP13 can regulate AKT phosphorylation by stabilizing PTEN protein (39). Thus, USP13 can suppress glycolysis and cell proliferation. miR-135b could promote AKT phosphorylation and enhance glycolysis in Caco2 and HCT-116 CRC cells with wild-type PTEN (45). PTEN inactivation by mutation or allelic loss also occurs in CRCs. CO-115 CRC cells have mutations leading to the premature stop codon of PTEN (45). In CO-115 cells, we found that miR-135b did not affect glucose uptake and glycolysis. Therefore, miR-135b promotes glucose uptake and glycolysis of CRC cells via the downregulation of PTEN. Moreover, miR-135b promoted the proliferation of HCT-116 CRC cells, but not with the restoration of USP13 or 
overexpression of PTEN. APC was proven to be a target of miR-135b and form a feedback loop with miR-135b (36). We also found that knockdown of USP13 upregulated the levels of endogenous miR-135b in the presence of wild-type PTEN. miR-135b can activate the expression of miR-135b itself by inhibiting the expression of USP13, thus attenuating the inhibitory effect of PTEN. These results reveal positive feedback loops between miR-135b and PTEN inactivation via USP13 in CRCs. Thus, this study provides support for the therapeutic potential of miR-135b in the targeted therapy of CRC.

\section{References}

1. Labianca R, Nordlinger B, Beretta GD, et al: Early colon cancer: ESMO Clinical Practice Guidelines for diagnosis, treatment and follow-up. Ann Oncol 24: vi64-vi72, 2013.

2. Paquin MC, Leblanc C, Lemieux E, Bian B and Rivard N: Functional impact of colorectal cancer-associated mutations in the transcription factor E2F4. Int J Oncol 43: 2015-2022, 2013.

3. Garcia Sanchez J: Colonoscopic polypectomy and long-term prevention of colorectal cancer deaths. Rev Clin Esp 212: 408, 2012 (In Spanish).

4. Nystrom M and Mutanen M: Diet and epigenetics in colon cancer. World J Gastroenterol 15: 257-263, 2009.

5. Kim YI: Nutritional epigenetics: impact of folate deficiency on DNA methylation and colon cancer susceptibility. J Nutr 135 2703-2709, 2005.

6. Yamashita K, Dai T, Dai Y, Yamamoto F and Perucho M: Genetics supersedes epigenetics in colon cancer phenotype. Cancer Cell 4: 121-131, 2003.

7. Markman B, Javier Ramos F, Capdevila J and Tabernero J: EGFR and KRAS in colorectal cancer. Adv Clin Chem 51: 71-119, 2010

8. Yu S, Xiao X, Lu J, Qian X, Liu B and Feng J: Colorectal cancer patients with low abundance of KRAS mutation may benefit from EGFR antibody therapy. PLoS One 8: e68022, 2013.

9. Yokota T: Are KRAS/BRAF mutations potent prognostic and/or predictive biomarkers in colorectal cancers? Anticancer Agents Med Chem 12: 163-171, 2012.

10. Hamilton SR: BRAF mutation and microsatellite instability status in colonic and rectal carcinoma: context really does matter. J Natl Cancer Inst 105: 1075-1077, 2013.

11. Tonini G, Imperatori M, Vincenzi B, Frezza AM and Santini D Rechallenge therapy and treatment holiday: different strategies in management of metastatic colorectal cancer. J Exp Clin Cancer Res 32: 92, 2013 .

12. Fodde R: The APC gene in colorectal cancer. Eur J Cancer 38: 867-871, 2002

13. Woodford-Richens KL, Rowan AJ, Gorman P, et al: SMAD4 mutations in colorectal cancer probably occur before chromosomal instability, but after divergence of the microsatellite instability pathway. Proc Natl Acad Sci USA 98: 9719-9723, 2001

14. de Weger VA, Turksma AW, Voorham QJ, et al: Clinical effects of adjuvant active specific immunotherapy differ between patients with microsatellite-stable and microsatellite-instable colon cancer. Clin Cancer Res 18: 882-889, 2012.

15. Berg M, Danielsen SA, Ahlquist T, et al: DNA sequence profiles of the colorectal cancer critical gene set KRAS-BRAFPIK3CA-PTEN-TP53 related to age at disease onset. PLoS One 5: e13978, 2010 .

16. Ilyas M, Tomlinson IP, Rowan A, Pignatelli M and Bodmer WF: Beta-catenin mutations in cell lines established from human colorectal cancers. Proc Natl Acad Sci USA 94: 10330-10334, 1997.

17. Georgescu MM: PTEN tumor suppressor network in PI3K-Akt pathway control. Genes Cancer 1: 1170-1177, 2010.

18. Song MS, Salmena L and Pandolfi PP: The functions and regulation of the PTEN tumour suppressor. Nat Rev Mol Cell Biol 13: 283-296, 2012

19. Nassif NT, Lobo GP, Wu X, et al: PTEN mutations are common in sporadic microsatellite stable colorectal cancer. Oncogene 23 : 617-628, 2004

20. Qin Q, Furong W and Baosheng L: Multiple functions of hypoxia-regulated miR-210 in cancer. J Exp Clin Cancer Res 33 $50,2014$.
21. Wang $\mathrm{Y}$ and Taniguchi T: MicroRNAs and DNA damage response: implications for cancer therapy. Cell Cycle 12: 32-42, 2013.

22. Krishnan K, Steptoe AL, Martin HC, et al: MicroRNA-182-5p targets a network of genes involved in DNA repair. RNA 19: 230-242, 2013

23. He YQ, Sheng JQ, Ling XL, et al: Estradiol regulates miR-135b and mismatch repair gene expressions via estrogen receptor-beta in colorectal cells. Exp Mol Med 44: 723-732, 2012.

24. Ma L, Teruya-Feldstein $\mathbf{J}$ and Weinberg RA: Tumour invasion and metastasis initiated by microRNA-10b in breast cancer. Nature 449: 682-688, 2007.

25. Pencheva $\mathrm{N}$ and Tavazoie SF: Control of metastatic progression by microRNA regulatory networks. Nat Cell Biol 15: 546-554, 2013.

26. Rottiers V and Näär AM: MicroRNAs in metabolism and metabolic disorders. Nat Rev Mol Cell Biol 13: 239-250, 2012.

27. Dumortier O, Hinault C and Van Obberghen E: MicroRNAs and metabolism crosstalk in energy homeostasis. Cell Metab 18: 312-324, 2013

28. Zhai $\mathrm{H}$ and $\mathrm{Ju} \mathrm{J}$ : Implications of microRNAs in colorectal cancer development, diagnosis, prognosis, and therapeutics. Front Genet 2: 78, 2011.

29. Dong Y, Wu WK, Wu CW, Sung JJ, Yu J and Ng SS: MicroRNA dysregulation in colorectal cancer: a clinical perspective. Br J Cancer 104: 893-898, 2011.

30. Schetter AJ, Okayama $\mathrm{H}$ and Harris CC: The role of microRNAs in colorectal cancer. Cancer J 18: 244-252, 2012.

31. Brunet Vega A, Pericay C, Moya I, et al: microRNA expression profile in stage III colorectal cancer: Circulating miR-18a and miR-29a as promising biomarkers. Oncol Rep 30: 320-326, 2013.

32. Khatri R and Subramanian S: MicroRNA-135b and its circuitry networks as potential therapeutic targets in colon cancer. Front Oncol 3: 268, 2013.

33. Wu CW, Ng SC, Dong Y, et al: Identification of microRNA-135b in stool as a potential noninvasive biomarker for colorectal cancer and adenoma. Clin Cancer Res 20: 2994-3002, 2014.

34. Wu W, Wang Z, Yang P, et al: MicroRNA-135b regulates metastasis suppressor 1 expression and promotes migration and invasion in colorectal cancer. Mol Cell Biochem 388: 249-259, 2014.

35. Munding JB, Adai AT, Maghnouj A, et al: Global microRNA expression profiling of microdissected tissues identifies miR-135b as a novel biomarker for pancreatic ductal adenocarcinoma. Int $\mathbf{J}$ Cancer 131: E86-E95, 2012.

36. Valeri N, Braconi C, Gasparini P, et al: MicroRNA-135b promotes cancer progression by acting as a downstream effector of oncogenic pathways in colon cancer. Cancer Cell 25: 469-483, 2014.

37. Lee JT, Shan J, Zhong J, et al: RFP-mediated ubiquitination of PTEN modulates its effect on AKT activation. Cell Res 23: $552-564,2013$.

38. Wang X, Trotman LC, Koppie T, et al: NEDD4-1 is a proto-oncogenic ubiquitin ligase for PTEN. Cell 128: 129-139, 2007.

39. Zhang J, Zhang P, Wei Y, et al: Deubiquitination and stabilization of PTEN by USP13. Nat Cell Biol 15: 1486-1494, 2013.

40. Donohoe DR, Collins LB, Wali A, Bigler R, Sun W and Bultman SJ: The Warburg effect dictates the mechanism of butyrate-mediated histone acetylation and cell proliferation. Mol Cell 48: 612-626, 2012.

41. Najafov A and Alessi DR: Uncoupling the Warburg effect from cancer. Proc Natl Acad Sci USA 107: 19135-19136, 2010.

42. Faltejskova P, Bocanek O, Sachlova M, et al: Circulating miR-17-3p, miR-29a, miR-92a and miR-135b in serum: evidence against their usage as biomarkers in colorectal cancer. Cancer Biomark 12: 199-204, 2012.

43. Xu XM, Qian JC, Deng ZL, et al: Expression of miR-21, miR-31, miR-96 and miR-135b is correlated with the clinical parameters of colorectal cancer. Oncol Lett 4: 339-345, 2012.

44. Zhao Y, Butler EB and Tan M: Targeting cellular metabolism to improve cancer therapeutics. Cell Death Dis 4: e532, 2013.

45. Ahmed D, Eide PW, Eilertsen IA, et al: Epigenetic and genetic features of 24 colon cancer cell lines. Oncogenesis 2: e71, 2013. 